\title{
Cintia Pinillos
}

Cintia Pinillos es docente e investigadora de la Universidad Nacional de Rosario y de la Universidad Nacional de Entre Ríos, Argentina.

E-mail: cintiapinillos@hotmail.com

\section{resumen}

El presente trabajo propone una reflexión sobre el aporte que la producción académica de Guillermo O'Donnell ha realizado a la Ciencia Política en general y a la política comparada en particular. En este sentido, pone el énfasis en su capacidad para iniciar agendas de investigación que fueron centrales para el desarrollo de estudios comparados sobre procesos latinoamericanos recientes. Asimismo, se menciona su contribución en términos de innovación conceptual para comprender tanto el autoritarismo como la democratización en contextos diversos.

\section{palabras clave}

Guillermo O’Donnell / política comparada / América latina / innovación conceptual / democratización

\section{summary}

This work aims to consider the contribution of the academic production of Guillermo O'Donnell to Political Science in general and to Comparative Plitics in particular. In that respect, the article emphasizes his ability to lead investigation agendas which were essential in the development of comparative studies about recent Latin American processes. Likewise, the paper specially highlights his contribution in terms of conceptual innovation to understand not only the authoritarianism but also the democratization in different contexts. 
Cuando desde la Facultad de Ciencia Política y Relaciones Internacionales de la Universidad Nacional de Rosario comenzamos a pensar en la Jornada de Homenaje a Guillermo O'Donnell sabíamos que esta actividad formaría parte de una serie de recordatorios y homenajes que se realizarían en distintos lugares de nuestro país y del mundo, promovidos por prestigiosas instituciones académicas. Sin embargo, consideramos que nuestro homenaje debía contribuir a promover la reflexión sobre el aporte de O'Donnell a la Ciencia Política y la relevancia de su legado para las actuales y futuras generaciones de politólogos en el marco de nuestra comunidad académica.

A esta razón inicial que daba fundamento a la convocatoria, se sumaban otras que lo hacían necesario en un sentido aún más profundo. Guillermo O’Donnell mantuvo hasta el final relaciones de amistad duraderas con colegas de nuestra institución. Con algunos de ellos produjo en colaboración trabajos académicos valiosos. A la par de esas relaciones personales de afecto y cooperación intelectual, O’Donnell fue construyendo a lo largo del tiempo un vínculo comprometido con nuestra institución y sobre todo en los últimos años participó de distintas actividades que favorecieron el diálogo con docentes y estudiantes.

Por mi parte, no tuve la fortuna de conocer a Guillermo O'Donnell en profundidad como gran parte de los expositores que participaron de la Jornada de Homenaje. Lo conocí a través de su obra, leyéndolo incansablemente y escuchándolo en distintas conferencias que ha dictado en reuniones científicas nacionales e internacionales. Más recientemente, en el marco del Seminario Internacional "Revisitando el concepto de democracia delegativa", realizado en nuestra Facultad a finales de 2009, tuve la posibilidad de intercambiar algunas palabras con él, y de confirmar su interés por vincularse con estudiantes de grado y posgrado, así como su atención al escuchar nuestras intervenciones y preguntas. Tal vez por las razones expuestas ese Homenaje y las notas que siguen no pertenecen al pasado sino al futuro de la Ciencia Política.

Durante muchos años Guillermo O’Donnell escribió sobre Argentina desde el exterior y reflexionó sobre América latina desde Estados Unidos. Sin embargo, sus trabajos nunca fueron extranjeros, ya que siempre estuvieron inspirados en un profundo conocimiento de nuestro país y de la región. Entre sus interlocutores estuvieron colegas formados en universidades norteamericanas y europeas, pero también colegas, discípulos y amigos de las universidades latinoamericanas. Él solía incluir en un mismo grupo a los autores nacidos en alguno de nuestros países y aquellos que sin ser de América latina, se habían dedicado seriamente a conocer los procesos políticos e hicieron contribuciones valiosas justamente porque intentaron entender los problemas latinoamericanos en sus propios términos (2007: 188). Los trabajos de Guillermo O'Donnell influyeron en los principales debates de la Ciencia Política sobre el autoritarismo y la democracia, pero siempre fueron escritos desde América latina.

O'Donnell es un politólogo internacional en todo el sentido de la palabra, por el tenor de su aporte y por el reconocimiento que ha cosechado a lo largo de su carrera. Aún así, el motor de su inquietud intelectual siempre fue la tortuosa historia de la democracia en América latina y sobre todo en nuestro país. 
El politólogo argentino ha dedicado buena parte de los últimos años de su vida académica a una tarea laboriosa de ordenamiento de su frondosa producción, favoreciendo nuestro acercamiento y el de las futuras generaciones a su legado. En este sentido, las compilaciones Contrapuntos. Ensayos escogidos sobre autoritarismo y democratización (1997), Disonancias (2007) y Catacumbas (2008), constituyen una puerta de ingreso a su obra, ya que además de reunir trabajos difundidos ampliamente en nuestro medio, se incluyen textos que habían tenido menor difusión. Esos libros son fundamentales, además, porque en ellos O'Donnell explicita el contexto de producción de cada trabajo. Con una prosa atrapante, en las introducciones de estos libros y en las notas al pie de los textos allí reunidos, nos abre la cocina de su producción, dejando pistas acerca de cómo fueron tomando forma aquellos problemas de investigación que luego han contribuido a iniciar importantes agendas de debate más allá de nuestras fronteras. En esos párrafos nos invita a reconstruir los viajes, a visitar las instituciones, a conocer a los interlocutores, a habitar los encuentros y desencuentros de una generación de científicos sociales que ha construido las bases de nuestra disciplina. En muchos casos, los trabajos compilados tienen como escenario los pasajes más oscuros y dolorosos de la historia reciente de nuestro país, y a partir de ellos se comprenden los dilemas, las tristezas y esperanzas que lo impulsaron a promover alrededor del mundo discusiones sobre la naturaleza del autoritarismo y la democracia.

La desaparición de Guillermo O'Donnell abre un espacio de recapitulación y nos obliga a reflexionar sobre nuestras propias prácticas científicas. Para quienes nos interesamos por la política comparada, es momento de volver detenidamente sobre su obra para reflexionar sobre lo que se está produciendo hoy en ese campo de la Ciencia Política desde (y sobre) América latina, en el mismo sentido en el que él lo entendía.

A diferencia de buena parte de la producción incluida en el sub campo de la política comparada contemporánea, el politólogo argentino recurría a la comparación para mostrar los límites de las generalizaciones disponibles inspirado en la evidencia que observaba en los procesos históricos, preocupado por los abusivos estiramientos conceptuales y los postulados estilizados que si bien tenían utilidad para incluir un número amplio y diverso de casos, poco podían explicar de la naturaleza profunda de los mismos.

Los trabajos de O'Donnell estaban guiados a la vez por un conocimiento profundo de los procesos históricos y la intención de promover explicaciones que pudieran trascender la realidad que originalmente lo había inspirado para poder analizar fenómenos semejantes. En este camino, desde su trabajo fundamental Modernización y autoritarismo (2011 [1972]) no dudó en discutir con las teorías predominantes cuando era necesario iluminar de manera más adecuada aquellos procesos que habían desencadenado fenómenos anclados temporal y espacialmente en nuestra región, para poder explicarlos y definir patrones que permitieran analizar casos semejantes.

El paradigma dominante en los '60 para estudiar la relación entre el desarrollo económico y la vigencia de la democracia política, había sido delineado por Lipset 
a comienzos de la década. Esta línea de investigación había iniciado una prolífica bibliografía comparada y parecía tener una validez prácticamente general, promoviéndose como uno de los aportes indiscutibles para abordar los procesos en curso en distintas regiones. Guillermo O'Donnell llamó "metáfora optimista" a esta generalización explicativa que rezaba que a más desarrollo socioeconómico había más probabilidades de que floreciera una democracia política. El politólogo argentino pudo identificar los límites de este aporte para explicar la política en Argentina y de otros países de América del Sur. Para él era necesario superar la fotografía y reconstruir la película, para comprender la dirección del movimiento de los actores y los patrones más frecuentes de interacción entre ellos (2011: 19). Recuperar el sentido de los procesos históricos de Argentina y Brasil, lo llevó a comprender la compleja naturaleza del autoritarismo burocrático en sociedades heterogéneas.

En los trabajos de O'Donnell la teoría es interrogada por los procesos históricos, que son en definitiva los que definen su destino. Ésta fue la premisa que guió su primer gran aporte a las Ciencias Sociales y que va a aparecer, encarnada en distintas estrategias y estilos a lo largo de toda su obra. Recuperando la clasificación propuesta por Theda Skocpol y Margaret Somers (1994), emerge como contraste de contextos para iluminar las diferencias y similitudes de las culturas políticas argentina y brasileña en Y a mí que mierda me importa. Notas sobre sociabilidady política en Argentina y Brasil ${ }^{1}$, o para inspirar e ilustrar su propuesta teórica alternativa en las diferentes versiones de su famoso concepto democracia delegativa. ${ }^{2}$

Aunque como O'Donnell nos cuenta, se pasó muchos años estudiando un tema que aborrecía, el autoritarismo, siempre su motivación central era encontrar los signos, los caminos, que conducirían a la democracia, o más claramente a la profundización de un proceso de democratización que trascendiera al régimen político e involucrara al Estado, a la sociedad, a la ciudadanía. Estas huellas lo llevaron a buscar en los movimientos de los actores y en la naturaleza de las instituciones que configuraban los procesos históricos respuestas más satisfactorias y precisas. Para la literatura comparada más encumbrada, las nuevas democracias que emergían en los países latinoamericanos en los ' 80 , nuestras democracias realmente existentes, diversas y esquivas desde los parámetros establecidos por el derrotero de las democracias noroccidentales, se encontraban salvo excepciones a mitad de camino de lo que las corrientes teóricas predominantes consideraban el punto de llegada, las poliarquías consolidadas. La literatura sobre la democratización promovida por O’Donnell propuso nuevos conceptos que, sin caer en la fácil tentación del parroquianismo, pudieran dar cuenta de la naturaleza de los procesos políticos en nuestra región en un nivel medio de generalidad.

En este sentido, la obra de O'Donnell, se inscribe en las principales agendas de la política comparada latinoamericana, y las enriquece a lo largo de más de cuatro décadas. Como afirma Munck, la teorización inspirada en América latina ha combinado información acerca de los casos y procesos políticos de la región con ideas de los autores clásicos de la teórica política y social. En este camino, se han producido conceptos nuevos o se han reformado los viejos, considerando varia- 
ciones empíricas entre los casos para ofrecer formulaciones iniciales a problemas sustantivos. Guillermo O'Donnell ha generado un rol relevante como promotor de agendas de estudio sobre la política en América latina. La mayor parte de la teorización puede ser caracterizada como de "alcance medio", concentrada en alguna dimensión de la política o en parte de un proceso más amplio (Munck, 2007: 9).

La insatisfacción de O'Donnell con las respuestas disponibles que intentaban explicar los procesos de democratización a partir de etapas inspiradas por procesos anteriores, queda claramente explicitada en Ilusiones sobre la consolidación (1996) y en un trabajo que fue publicado por primera vez hace más de una década: Teoría democrática y política comparada (2000). En el primer artículo mencionado aparece el germen del concepto de poliarquías institucionalizadas informalmente, que lo llevaría a producir una de las caracterizaciones más interesantes para comprender el funcionamiento de las instituciones políticas en nuestros países que, luego, se plasmaría en otros trabajos. En este artículo queda de manifiesto la incomodidad que le generaban las definiciones poco precisas que generaban limbos teóricos. El amplio alcance y la escasa efectividad que alcanzó en la década de los '90 el concepto de consolidación democrática, constituye un ejemplo de estrategias comparativas que si bien presentan una fuerte capacidad para analizar en contextos diversos, presentan serias dificultades para decir algo relevante sobre los casos que son analizados. Por su parte, Teoría democrática y política comparada, es definido por su propio autor como un texto sobre teoría democrática a secas, que persigue el objetivo de aclarar el terreno conceptual para futuros trabajos. Buscando herramientas teóricas más adecuadas que las disponibles para comprender las nuevas democracias, encuentra inconsistencias que no solo dificultaban la comprensión de muchos casos de reciente democratización sino que ponían en cuestión la propia consistencia de la teoría democrática (2000: 521). Así plantea que sería un error emprender el estudio comparativo como si las teorías disponibles ofrecieran un ancla conceptual firme (2000: 523). En este sentido, para O’Donnell el desafío no radicaba exclusivamente en evaluar la significación dispar de los adjetivos que se proponían para precisar el fenómeno democrático en distintos contextos, sino también en interpelar el propio campo semántico de los conceptos de democracia disponibles. Los argumentos de este trabajo y otros posteriores, decantarían luego en su último libro individual Democracia, agencia y estado. Teoría con intensión comparativa (2010), en el cual sintetiza sus aportes a la teoría democrática, recuperando su reflexión de varias décadas sobre la democracia.

Como todo buen investigador con una sólida formación teórica sabía que en el mismo acto de desarrollar empresas comparadas había que ocuparse adecuadamente de las "incertidumbres conceptuales".

En un artículo reciente, Aníbal Pérez Liñán (2008) nos recuerda que si bien comparamos fundamentalmente para probar hipótesis, hay otras buenas razones por las cuales se compara, entre ellas está la formación de conceptos y la formulación de hipótesis. Es a través de la observación comparativa que identificamos nuevas categorías para pensar el mundo, y la importancia de esta tarea se ve cla- 
ramente reflejada en aquellos conceptos innovadores que inauguraron debates y nuevos campos de investigación. Asimismo, podemos compartir con el autor que las proposiciones teóricas que realizan una mayor contribución suelen estar fundadas en un profundo conocimiento comparativo de al menos algunos casos. La literatura teórica contribuye sin dudas al proceso de formulación de hipótesis, pero la reflexión puramente teórica, sin el conocimiento de distintos casos históricos, puede resultar insuficiente (2008: 6).

O'Donnell fue un agudo productor de conceptos -Estado burocrático-autoritario, poliarquías informalmente institucionalizadas, democracia delegativa, entre otros- y sin dudas el vuelo que los mismos alcanzaron fue impulsado por el conocimiento comparativo que los habían inspirado.

Entiendo que los politólogos y las politólogas que desde América latina estamos produciendo en el campo de la política comparada tenemos que recordar, inspirados en el camino transitado por Guillermo O'Donnell, que es posible hacer contribuciones relevantes a la ciencia política partiendo de la especificidad histórica de nuestra región y que los estudios de área son una excelente estrategia para abordar problemas complejos y sortear las falsas promesas de relaciones causales estilizadas que no conducen a producir conocimiento significativo. Como ha demostrado el autor argentino, si nuestras investigaciones sobre un número modesto de casos que comparten aspectos semejantes y diferentes están bien formuladas e interpeladas teóricamente, pueden permitirnos identificar temas y problemas que, con características propias se desarrollan en otras partes del mundo.

Tomar este camino no implica lanzarnos a proponer conceptos e hipótesis parroquiales sino conocer en profundidad las teorías disponibles y a partir de ese conocimiento atrevernos a dudar cuando no nos ayuden a explicar nuestros casos de estudio. Tomar este camino implica no resignarnos a producir datos que cuadren adecuadamente en teorías extranjeras para ocupar un espacio en las notas al pie de los autores centrales de turno. Retomando el desafío de los mejores exponentes actuales y pasados de las ciencias sociales latinoamericanas, de las que sin dudas Guillermo O'Donnell es uno de los autores más importantes, tomar este camino nos impulsará a avanzar al mismo tiempo en la elaboración de datos comparables y en formulación de teoría. Como él enunciara, nos llevará a negarnos “... a reproducir en las ciencias sociales y en la historia, algo muy semejante a la ecuación de la dependencia originaria de América Latina" (2008: 188). ${ }^{3}$

\section{Referencias}

1. En Contrapuntos: ensayos escogidos sobre autoritarismo y democratización (1997).

2. Se sugiere consultar su último libro, recientemente publicado por Prometeo: O’Donnell, G., Iazzetta, O. y Quiroga, H. (coordinadores), 2011.

3. Originalmente escrito cuando recibió el premio Kalman Silvert. Congreso de LASA. Dallas, abril de 2003.

\section{Bibliografía}

T. SKOCPOL y M. SOMERS (1994), "The uses of comparative History in Macrosocial Inquiry", in 
Social Revolutions in de Third World, Cambridge, Cambridge University Press.

G. MUNCK (2007), "Agendas y estrategias de investigación en el estudio de la política latinoamericana", en Revista de Ciencia Politica, Vol 27, $\mathrm{N}^{\circ} 1$.

G. O’DONNELL (1996), "Ilusiones sobre la consolidación”, en Nueva Sociedad, №144. Julioagosto.

G. O’DONNELL (1997), Contrapuntos. Ensayos escogidos sobre autoritarismo y democratización, Buenos Aires, Paidós.

G. O’DONNELL (2000), “Teoría democrática y política comparada”, en Desarrollo Económico, Vol. $39, \mathrm{~N}^{\circ} 156$, enero-marzo.

G. O’DONNELL (2007), “Ciencias sociales en América Latina. Mirando hacia el pasado y atisbando el futuro", en Disonancias. Críticas democráticas a la democracia, Buenos Aires, Prometeo Libros.

G. O'DONNELL (2008), Catacumbas, Buenos Aires, Prometeo Libros.

G. O'DONNELL (2010), Democracia, agencia y estado. Teoría con intensión comparativa, Buenos Aires, Prometeo Libros.

G. O'DONNELL, O. IAZZETTA y H. QUIROGA (2011), Democracia delegativa, Buenos Aires, Prometeo Libros.

A. PÉREZ-LINÁN (2004), "Cuatro razones para comparar", en Boletín de Política Comparada, $\mathrm{N}^{\circ}$ 1 , junio.

Recibido 28/08/2012. Aceptado: 01/10/2012.

Cintia Pinillos, "El legado de Guillermo 0'Donnell a la política comparada latinoamericana". Revista Temas y Debates. ISSN 1666-0714, año 16, número 24, julio-diciembre 2012, pp. 67-73. 